\title{
Optimal Location and Size of SVC and TCSC for Multi-objective Static Voltage Stability Enhancement
}

\author{
R. Benabid ${ }^{1}$ and M. Boudour ${ }^{2}$ \\ ${ }^{1}$ Nuclear Center Research of Birine \\ B.P. 180, 17200, Djelfa (Algeria) \\ E-mail: rabah_benabid@yahoo.fr \\ ${ }^{2}$ Department of Electrical Engineering \\ University of Sciences \& Technology Houari Boumediene \\ El Alia, BP.32, Bab Ezzouar, 16111, Algiers (ALGERIA) \\ E-mail: mboudour@IEEE.org
}

\begin{abstract}
A Non-dominated Sorting Particle Swarm Optimization (NSPSO) is used to solve a mixed continuousdiscreet Multi-objective optimization problem witch consist of optimal location and size of Static Var Compensators (SVC) and Thyristor Controlled Series Capacitors (TCSC) in order to maximize Static Voltage Stability Margin (SVSM), reduce power losses (PL) and minimize load Voltage Deviations (VD). While finding the optimal location, thermal limits for the lines and voltage limits for the buses are taken as security constraints. The optimization is performed considering two and three objectives for various combinations of FACTS. Simulations are performed on IEEE 14 test system for optimal location and size of FACTS devices. The obtained results are very encouraging and reveal the capability of the method to generate well-distributed non-dominated Pareto front.
\end{abstract}

Keywords-Static voltage stability margin, SVC, TCSC, Multi-objective optimization, Non-dominated Sorting Particle Swarm Optimization.

\section{Introduction}

In the last few years, voltage collapse problems in power systems have been of permanent concern for electric utilities: several major blackouts throughout the world have been directly associated to this phenomenon, e.g. in France, Italy, Japan, Great Britain, WSCC in USA, etc. [1]. The analysis of this problem shows that the major causes is the system's inability to meet Var demands.

Several efforts have been made to find the ways to assure the security of the system in terms of voltage stability. It is found that flexible AC transmission system (FACTS) devices are a good choice to improve the SVSM in power systems, which operates near the steadystate stability limit and may result in voltage instability. Moreover it can provide benefits in increasing system transmission capacity and power flow control flexibility and rapidity [2]. Taking advantages of the FACTS devices depends greatly on how these devices are placed in the power system, namely on their location and size [3].

The optimal location and size of FACTS devices has retained the interest of worldwide researchers in power systems. In the stationary mode, FACTS devices are used to control the power flow in the transmission lines as well as the bus voltages. The required objectives can be of technical order or of an economic nature. Various mathematical methods and criteria are used to optimal allocation of these devices in the power systems [4]-[8].

A population based approach's named heuristic method retained the interest of several researchers. Malihe et al. [3] use Particle Swarm Optimization (PSO) and Genetic Algorithms (GA) for planning SVC in order to enhance voltage profile and to reduce total real power losses. The two objectives are considered as the inputs of the fuzzy inference system and the output is an index of satisfaction of objectives. In [2] the PSO technique is used to find the optimal location of multi-type of FACTS devices, namely SVC, TCSC, and UPFC with minimum cost of installation and to improve the system loadability. The two objectives are converted into a single objective function. Other works in this field are presented in [9][11].

From the previous works, we can conclude that the problem of optimal location of FACTS devices is generally formulated as a mono-objective optimization problem that optimize a single objective function or transform several objectives to a single objective by aggregating or via a fuzzy inference system.

The formulation of optimal location of FACTS as multi-objective optimization problem is a new attempt in this field, the authors in [12], use a Multi-objective Particle Swarm Optimization (MOPSO) Algorithm to find the optimal location of Thyristor Controlled Series Compensator (TCSC) and its parameters in order to increase the Total Transfer Capability (TTC), reduce total transmission losses and minimize voltage deviation.

This paper investigates the optimal location of FACTS devices as a real multi-objective optimization 
problem. So, we used a Non-dominated Sorting particle Swarm Optimization (NSPSO) method to find the optimal size and placement of the two popular FACTS namely: TCSC and SVC considering different objectives such as increasing SVSM, decreasing $P L$, minimizing the load $V D$. The optimization procedure is performed for two up to four functions for single-type devices (one type of FACTS is considered) and multi-type of FACTS (both SVC and TCSC are considered). The optimized parameters of FACTS are the location and size for singletype case, plus the type of FACTS for multi-type optimization case.

Firstly, the problem is formulated as bi-objective optimization problem, considering only the minimization of real power losses and the maximization of SVSM. In the second step, three objectives are optimized, considering also, the minimization of load voltage deviation.

This paper is organized as follows: section 2 presents a brief introduction of multi-objective optimization problems. In section 3 the NSPSO algorithm is presented along with a detailed discussion. The FACTS modelling and problem formulation are presented in section 4. Finally, major contributions and conclusions are summarized in section 7 .

\section{Multi-objective Optimization Overview}

Many real-world problems involve simultaneous optimization of several objective functions. Generally, these functions are non-commensurable and often conflicting objectives. Multi-objective optimization with such conflicting objective functions gives rise to a set of optimal solutions, instead of one optimal solution. The reason for the optimality of many solutions is that no one can be considered to be better than any other with respect to all objective functions. These optimal solutions are known as Pareto-optimal solutions [13].

A general multi-objective optimization problem consists of a number of objectives to be optimized simultaneously and is associated with a number of equality and inequality constraints. It can be formulated as follows [13]:

$$
\begin{gathered}
\text { Minimize } f_{i}(x), i=1, \ldots, N_{o b j} \\
\text { Subject to constraints: }\left\{\begin{array}{rr}
g_{j}(x)=0 & j=1, \ldots, M \\
h_{k}(x) \leq 0 & k=1, \ldots K
\end{array}\right.
\end{gathered}
$$

where, $f_{\mathrm{i}}$ is the $i^{\text {th }}$ objective function; $x$ is the decision vector representing a solution, and $N_{o b j}$ is the number of objectives. To compare candidate solutions in multiobjective optimization problems, the concepts of Pareto dominance is used. A decision vector $u$ is said to dominate another vector $v$ (denoted $u<v$ ) if:

$$
f_{i}(u) \leq f_{i}(v) \wedge \exists i \in\{1,2, \ldots N\}: f_{i}(u) \prec f_{i}(v)
$$

In this case, the solution $u$ dominates $v ; u$ is called the non-dominated solution. The solutions that are nondominated within the entire search space are denoted as
Pareto-optimal and constitute the Pareto-optimal set or the Pareto-optimal front.

\section{Non-Dominated Sorting Particle Swarm Optimization Method}

There are several papers proposed to extend the Particle Swarm Optimization (PSO) method to handle a Multi-objective optimization problem [14-20]. Among these algorithms, NSPSO algorithm is based on the same non-dominated sorting concept used in NSGA-II [20]. This approach will ensure more non-dominated solutions can be discovered through the domination comparison operations. NSPSO is presented in detail bellow.

The figure 1 presents the principle of pbest selection proposed by NSPSO algorithm.

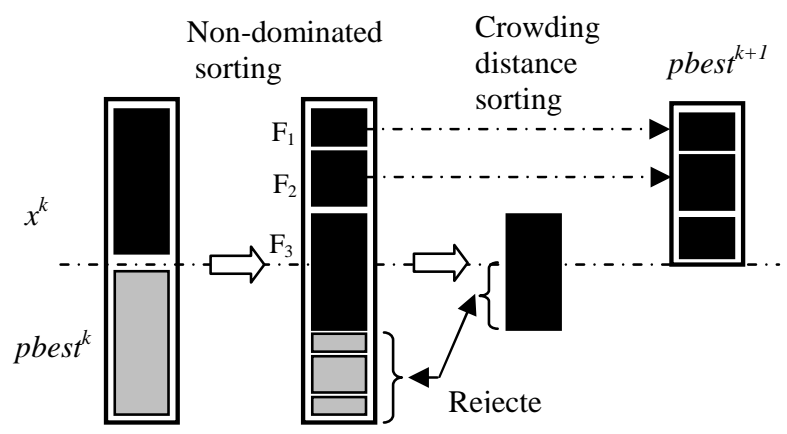

Fig.1. Principle of pbest selection proposed by NSPSO algorithm.

Instead of comparing solely on a particle's personal best with its potential offspring, the entire population of $N$ particles' personal bests and $N$ of these particles' offspring are first combined to form a temporary population of $2 \mathrm{~N}$ particles. After this, the non-dominated sorting concept is applied, where the entire population is sorted into various non-domination fronts. The first front being completely a non-dominant set in the current population and the second front being dominated by the individuals in the first front only and the front goes so on. Each individual in each front is assigned fitness values or based on front in which they belong to. Individuals in the first front are given a fitness value of 1 and individuals in second are assigned a fitness value of 2 and so on. In addition to the fitness value, a new parameter called crowding distance is calculated for each individual for ensure the best distribution in the solution. The crowding distance is a measure of how close an individual is to neighbors. The global best gbest $_{\mathrm{i}}$ for the $\mathrm{i}^{\text {th }}$ particle $x_{i}$ is selected randomly from the top part of the first front (the particles witch has the highest crowding distance). $N$ particles are selected based on fitness and the crowding distance to plays the role of pbest. Such as, when the first front has more than $N$ particles, we select the particles that have the highest distance. The update of the particles position in the research space is based on the two famous equations [21].

$$
\begin{gathered}
x_{i}^{k+1}=x_{i}^{k}+v_{i}^{k+1} \\
v_{i}^{k+1}=w v_{i}^{k}+c_{1} \text { rand }_{1} \times\left(\text { pbest }_{i}-x_{i}^{k}\right)+c_{2} \text { rand }_{2} \times\left(\text { gbest }_{i}-x_{i}^{k}\right)
\end{gathered}
$$


where,

$w \quad$ : weighting function,

$c_{j} \quad$ : weighting factor,

rand : random number between 0 and 1 ,

pbest $_{i}$ : personal best of the particle $i$,

gbest $t_{i}$ global best of the particle $i$,

$v_{i}{ }^{k} \quad$ : current velocity of agent $i$ at iteration $\mathrm{k}$,

$v_{i}^{k+1}$ : current velocity of agent $i$ at iteration

$x_{i}^{k} \quad$ : current position of agent $i$ at iteration $\mathrm{k}$,

$x_{i}^{k+1}$ : current position of agent $i$ at iteration $\mathrm{k}+1$.

The following weighting function is usually utilized [21]:

$$
w=w_{\max }-\frac{w_{\max }-w_{\min }}{\text { iter }_{\max }} \times \text { iter }
$$

where,

$$
\begin{array}{ll}
w_{\max } & \text { : initial weight, } \\
w_{\min } & : \text { final weight, } \\
\text { iter }_{\max } & : \text { maximum iteration number, } \\
\text { iter } & : \text { current iteration number. }
\end{array}
$$

The steps of basic NSPSO algorithm is presented as follow:

For each iteration $\mathrm{k}$ do:

1. $R^{k}=x^{k} \cup$ pbest $^{k}$ (combine the current solution and all personal best)

2. $F=n o n_{-}$dom_sort $\left(R_{t}\right)$ (Application the nondominated sorting on $R_{t}$ )

3. pbest $^{k+1}=\phi \& i=1$

4. until $\mid$ pbest $^{k+1}|+| F_{i} \mid \leq N$ (until the pbest set is filled)

a. $i=i+1$

$b$. Calculate the crowding distance for each particle in $F_{i}$

c. pbest $^{k+1}=$ pbest $^{k+1} \cup F_{i}$

5. Sort $(F i)$ (sort in descending order)

6. Select randomly gbest for each particle from a specified top part (e.g. top 5\%) of the first front $F_{1}$

7. $\mid$ pbest $^{k+1}|=|$ pbest $^{k+1} \mid \cup F_{i}\left(N-\mid\right.$ pbest $\left.^{k+1} \mid\right)$

(Choose the first $N-\mid$ pbest $\mid$ elements of $F_{i}$ )

8. $x^{k+1}$ (use (4) and (5) to calculate the new positions of particle with using the new pbest and gbest.

- $k=k+1$

\section{FACTS Design and Location}

As we already mentioned this paper focuses on the optimal location and design of two kinds of FACTS, namely the SVC and the TCSC. The model of these FACTS used in this paper is presented in detail bellow.

\section{A. Model of SVC}

The SVC is defined as a shunt connected static Var generator or consumer whose output is adjusted to exchange capacitive or inductive so as to maintain or control specific parameters of electrical power system, typically a bus voltage [22]. Like the TCSC, the SVC combines a series capacitor bank shunted by thyristor controlled reactor. In this paper, the SVC is considered as a synchronous compensator modeled as $P V$ bus, with $Q$ limits.

\section{B. Model of TCSC}

TCSC is a series compensation component which consists of a series capacitor bank shunted by thyristor controlled reactor. The basic idea behind power flow control with the TCSC is to decrease or increase the overall lines effective series transmission impedance, by adding a capacitive or inductive reactive correspondingly. The TCSC is modeled as variable impedance, where the equivalent reactance of line $X_{i j}$ is defined as:

$$
X_{i j}=X_{\text {line }}+X_{T C S C}
$$

where, $X_{\text {line }}$ is the transmission line reactance, and $X_{T C S C}$ is the TCSC reactance. The level of the applied compensation of the TCSC usually varies between $20 \%$ inductive and $80 \%$ capacitive [22].

\section{Problem formulation}

The optimal location and design of SVC and TCSC is formulated as mixed continues-discrete multi-objective optimization problem. The objectives considered in this paper are presented in detail below.

\section{1) Static Voltage Stability Margin (SVSM)} Static Voltage stability Margin (SVSM) or loading margin is the most widely accepted index for proximity of voltage collapse. The SVSM is calculated using Power System Analysis Toolbox (PSAT) [23]. SVSM is defined as the largest load change that the power system may sustain at a bus or collective of buses from a well defined operating point (base case). The maximization of SVSM can be presented as follows:

$$
\operatorname{Max}\{\operatorname{SVSM}\}
$$

\section{2) Real power losses (PL)}

This objective consists of minimizing the real power loss in the transmission lines and which can be expressed as:

$$
\operatorname{Min}\left\{\sum_{k=1}^{n l} g_{k}\left[V_{i}^{2}+V_{i}^{2}-2 V_{i} V_{j} \cos \left(\delta_{i}-\delta_{i}\right)\right]\right\}
$$

where, $n l$ is the number of transmission lines; $g_{k}$ is the conductance of the $k$ th line; $V_{i} \angle \delta_{i}$ and $V_{j} \angle \delta_{j}$ are the voltages at the end buses $i$ and $j$ of the $k^{\text {th }}$ line, respectively.

3) Voltage deviation (VD)

This function is to minimize the deviations in voltage magnitudes at load buses that can be expressed as: 


$$
\operatorname{Min}\left\{\sum_{k=1}^{N L}\left|V_{k}-V_{k}^{r e f}\right|\right\}
$$

where, $N L$ is the number of load buses; $V_{k}^{\text {ref }}$ is the prespecified reference value of the voltage magnitude at the $k$ th load bus. $V_{k}^{\text {ref }}$ is usually set to $1.0 \mathrm{pu}$.

\section{4) Equality and Inequality Constraints}

The equality and inequality constraints should be respected during the optimization procedure. The equality constraints represent the typical load flow equations. The inequality constraints represent the operating limits of the TCSC and SVC. Moreover, two security limits are considered in this paper, namely the thermal limits of the transmission lines and the bus voltage limits, which are applied on the two last objectives only ( $P L$ and $V D)$, because, in the general case, the voltage collapse occurs after the security limits have been exceeded.In this paper, if the security limits are not respected the current solution is rejected.

\section{Results and Discussions}

The proposed approach is applied on IEEE 14-bus test system [23]. The system consists of 14 buses, 20 lines, two generators, located at bus 1 and 2 , three synchronous compensators used only for reactive power support at buses 3, 6 and 8, and three transformers in lines 5-6, 4-9 and 4-7. The generators are modeled as $P V$ buses with $Q$ limits; the loads are typically represented as constant $P Q$ loads. In this paper, the increase in the load is regarded as the parameter which leads the power system to a voltage collapse.

$$
\left\{\begin{array}{l}
P_{L}=\lambda P_{0 L} \\
Q_{L}=\lambda Q_{0 L}
\end{array}\right.
$$

where, $P_{0 L}$ and $Q_{0 L}$ are the active and reactive base loads, whereas $P_{L}$ and $Q_{L}$ are the active and reactive loads at a bus $L$ for the current operating point. The load power factor is maintained constant during the load increasing.

The decision variables considered are the location and size of TCSC and SVC. The number of FACTS to be installed is chosen one for each type; also the limits are fixed at the beginning by the user. The reactance of TCSC is considered as a capacitive reactance varying continuously between $10 \%$ and $80 \%$ of the line reactance. The placement of TCSC is considered as a discreet variable, where all lines of the system (20 lines) are selected to be the optimal location of TCSC. The same thing for the SVC, which is considered as a synchronous compensator with a reactive power changing continuously between $0.1 \mathrm{pu}$ and 2 pu. The optimal location of SVC is, also, considered as a discreet decision variable, where all load buses are selected to be the optimal location of SVC.

In this paper, the optimal location and size of SVC and TCSC is performed for two multi-objective problems, considering several combinations of FACTS devices.

\section{A. Power losses and Voltage stability margin}

At first, we only considered two objective functions namely: PL and SVSM, the aim is to find the Pareto front which consists of optimal size and location of TCSC and SVC that maximize the SVSM and minimize PL. for all optimization cases, the number of population is fixed at 100 , and the number of generation is fixed at 120 .

Figure 2 depicts the non-dominated solution of optimal location and size of SVC. NSPSO provides 7 non-dominated solutions summarized in Table1.

We can conclude that buses $4,10,9,5,7$ and 14 are considered as best locations of SVC with different size. From these results, the decision maker (DM) can choose the optimal location to install the SVC: If the SVSM is preferred to $P L$, the DM could choose the bus number 9 as the optimal location of SVC with 2 pu of size (200 MVar). Whereas if PL is a priority, a SVC of $0.1 \mathrm{pu}$ of size installed at the bus 4 would be the optimal choice. Generally, the DM can choose other solutions from the non-dominated solutions according to the company policy.

Figure 3 depicts the non-dominated solution for the optimal location and size of TCSC considering the maximization of SVSM and the minimization of $P L$.

Figure 3 presents 522 non-dominated solutions of optimal location and size of TCSC. All 269 solutions indicate that the line 14 (bus 1-bus5) as the optimal location of TCSC with different size. The remainder set of solutions (253 solutions) indicate that the optimal placement of TCSC is the line 11 (bus 1-bus2). In the case where the SVSM is priority than the $P L$ objective, the DM will choose line 11 as the optimal location of TCSC of $80 \%$ of compensation level. This later provides the SVSM of $1.8830 \mathrm{pu}$. In the case where the $P L$ objective is priority than SVSM, the optimal location and size of TCSC is respectively line 14 and $22.7 \%$ of compensation level, where the $P L$ is $0.1346 \mathrm{pu}$.

Figure 4 depicts the non-dominated solutions of optimal location and size of both SVC and TCSC (the two FACTS are simultaneously optimized). Actually, the obtained solutions are the best combinations or the best coordination of SVC and TCSC. In this case, NSPSO provides 186 non-dominated solutions, where the installation of SVC of 2 pu size at the bus number 9, and the TCSC at the line 11 (bus1-bus2) with $80 \%$ compensation level provides the best SVSM of 2.4662 pu. Wheras, the installation of SVC of $0.1 \mathrm{pu}$ size at the bus number 5, and the TCSC at the line 12 (bus3-bus2) with $30.12 \%$ of compensation level provides the best $P L$ of $0.1355 \mathrm{pu}$.

\section{B. Static Voltage Stability Margin, Power Losses, and Voltage Deviation}


This case is more complicated than the previous one, where three objectives are considered namely: SVSM, $P L$, and $V D$. The aim is to optimize the location and the size of TCSC, and SVC witch maximize the SVSM and minimize the $P L$ and $V D$. The optimization is performed for single-type and multiple-type of FACTS.

Figure 4 presents 210 non-dominated solutions for the optimal location and size of SVC. Where, if $P L$ is priority, the optimal location of SVC of 0.1 pu size is the bus number 4 . Otherwise, if the SVSM is priority than other objectives, SVC of $2 \mathrm{pu}$ of size installed at bus number 9 is the optimal choice, in the case, where the $V D$ is priority, SVC of $0.1948 \mathrm{pu}$ of size installed at the bus number 13 is the optimal choice. Fig. 4 presents 888 nondominated solutions for the optimal locations and sizes of TCSC, where the most repeated solutions are line 11 is repeated 679 times and line 14 is repeated 175 times. In the case where, $P L$ is priority than other objectives, the optimal location of TCSC of $22.76 \%$ of compensation level installed at line 14. Otherwise, if the SVSM is priority, TCSC of $80 \%$ of compensation level installed at line 11 is the optimal choice, in the case, where the $V D$ is priority, the optimal solution the same for the case of SVSM. Fig. 5 presents 427 non-dominated solutions, where the installation of SVC of 2 pu of size at bus 9 and TCSC of $80 \%$ of level of compensation at line 11 gives the best SVSM. Whereas, the installation of SVC of 0.1 pu of size at bus 5 , and the TCSC of $25.55 \%$ of level of compensation at line 12 gives best value of $P L$. Finally the installation of SVC of size of 19.45 pu at bus 13 , and TCSC of $25.4 \%$ of level of compensation at line 8 gives the best value of $V D$.

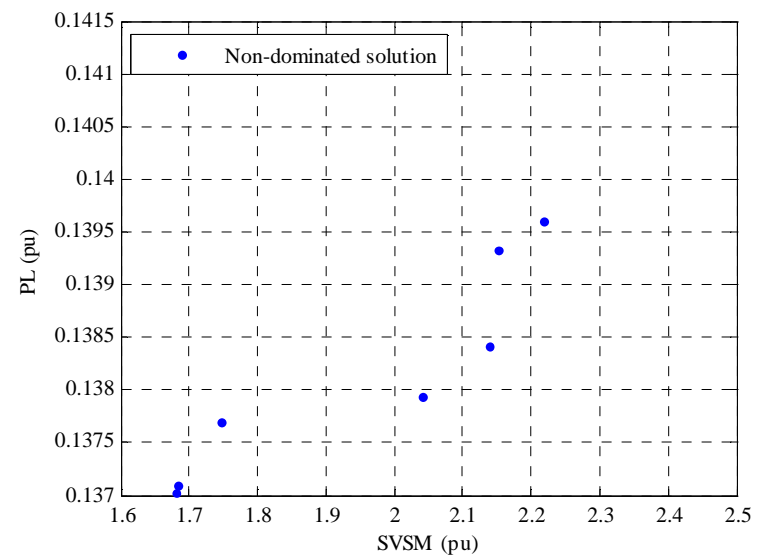

Fig. 2. Optimal location and size of SVC for two objectives.

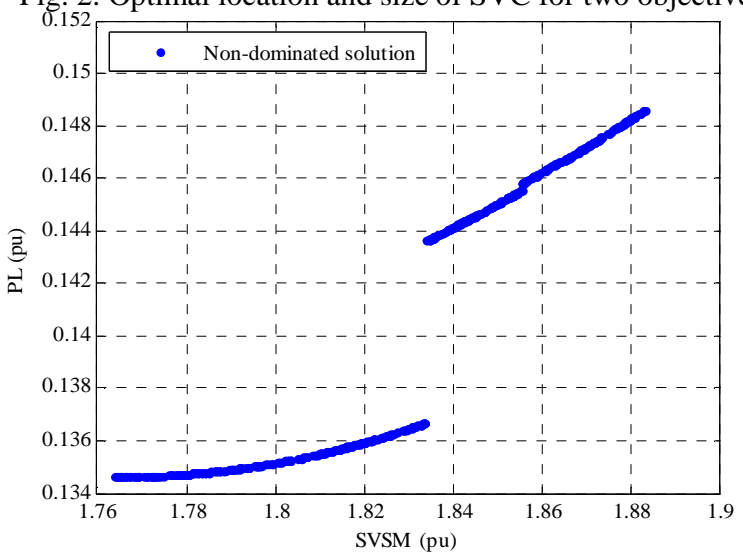

Fig. 3. Optimal location and size of TCSC for two objectives.

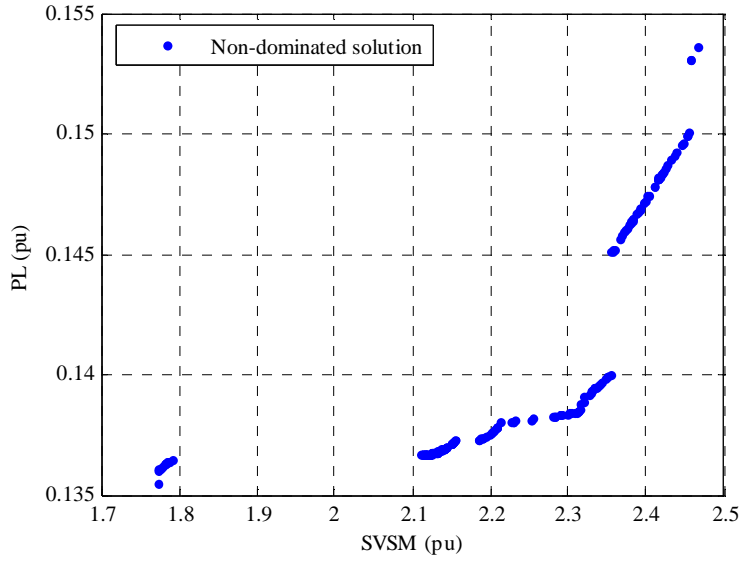

Fig. 4. Optimal location and size of SVC and TCSC for two objectives.

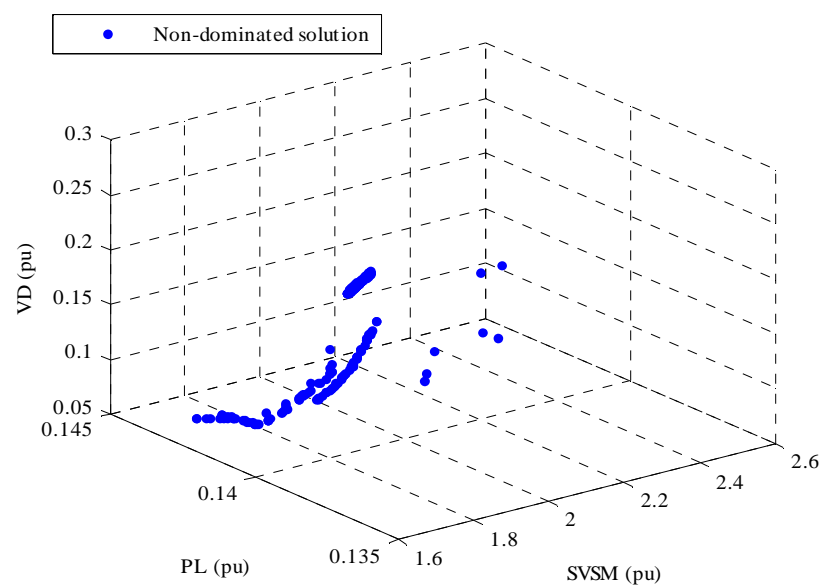

Fig. 5. Optimal location and size of SVC for three objectives.

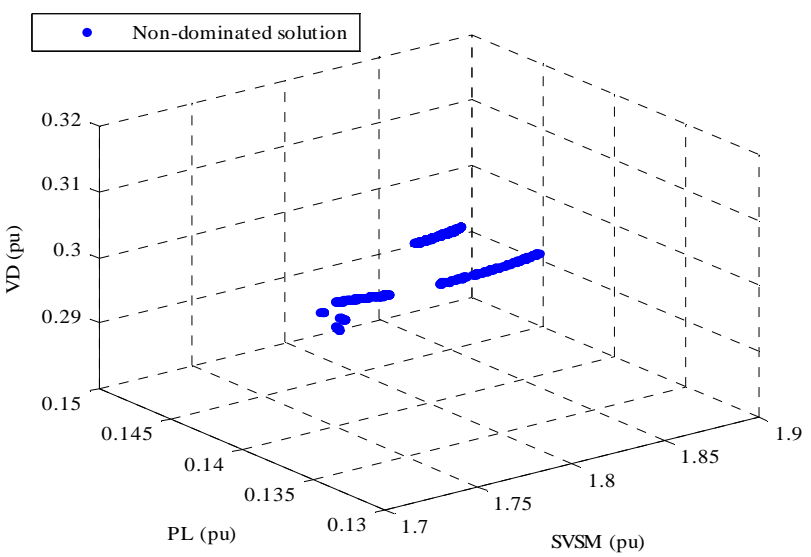

Fig. 6. Optimal location and size of TCSC for three objectives.

TABLE I-Non-dominated solutions for optimal location of SVC

\begin{tabular}{cccc}
\hline Bus & Size $(\mathrm{pu})$ & SVSM $(\mathrm{pu})$ & PL $(\mathrm{pu})$ \\
\hline 4 & 2.0000 & 2.1378 & 0.1384 \\
10 & 1.9236 & 2.1528 & 0.1393 \\
9 & 2.0000 & 2.2183 & 0.1396 \\
5 & 0.1000 & 1.6827 & 0.1371 \\
4 & 0.1000 & 1.6806 & 0.1370 \\
7 & 0.1000 & 1.7462 & 0.1377 \\
\hline 14 & 1.5997 & 2.0415 & 0.1379 \\
\hline
\end{tabular}




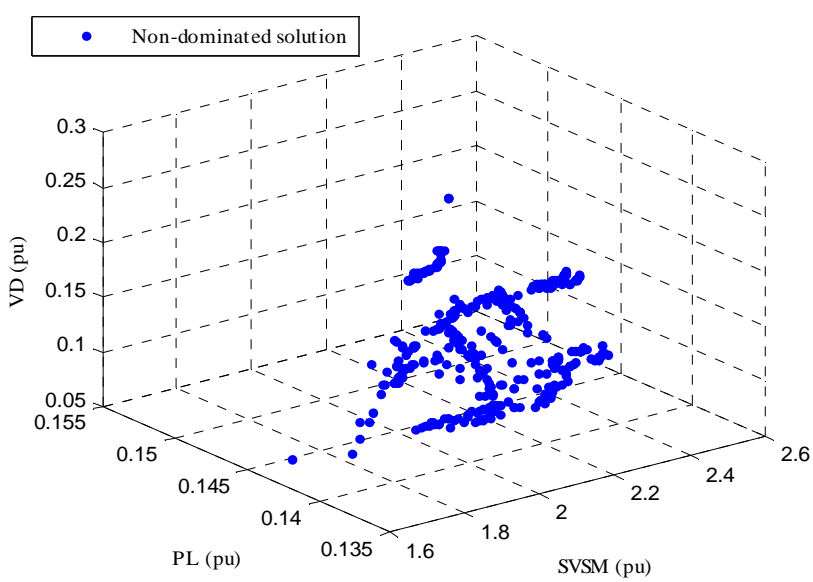

Fig. 7. Optimal location and size of SVC and TCSC for three objectives.

\section{Conclusion}

In this work, the optimal location and size of SVC and TCSC devices is found to maximize the SVSM, reduce the $P L$ and minimize $V D$. The problem is formulated as a mixed discreet-continuous multiobjective optimization problem. Simulations performed on IEEE 14-bus test system indicate that the proposed method is able to provide the optimal locations and sizes of multi-type of FACTS to be used by the DM in different planning studies to voltage stability improvement. Moreover, we can mention also, that the proposed method does not impose any limitation on the number of objectives to be optimized.

\section{References}

[1] A. Kazemi, and B. Badrzadeh, "Modeling and Simulation of SVC and TCSC to Study their Limits on Maximum Loadability Point," Electrical Power and Energy Systems, Vol. 26, pp. 619-626, Apr. 2004.

[2] M. Saravanan et al. "Application of particle swarm optimization technique for optimal location of FACTS devices considering cost of installation and system loadability", Electric Power System Research 77 (2007) 276-283.

[3] Malihe M. Farsangi, Hossien Nezamabadi-Pour, and K. Y. Lee, "Multi-objective VAR Planning with SVC for a Large Power System Using PSO and GA," Power System Conference \& Exposition, pp. 274-279, 2006.

[4] Y. Mansour et al. "SVC Placement Using Critical Modes of Voltage Instability", IEEE Transactions on Power Systems, Vol. 9, No. 2, pp.757-763, May 1994

[5] L.A.S. Pilotto, "Determination of Needed Facts Controllers That Increase Asset Utilization of Power Systems", IEEE Transactions on Power Delivery, Vol. 12, No 1, pp. 364-371, January 1997.

[6] S.N. Singh, "Placement of Facts Devices in Open Power Market", Proceedings of the 5th International Conference on Advances in Power System Control, Operation and Management, APSCOM 2000, pp. 173-177, Hong Kong, October 2000.

[7] M. K. Verma, "Optimal Placement of SVC for Static and Dynamic Voltage Security Enhancement", International Journal of Emerging Electric Power Systems, Vol. 2, Issue 2, Article 1050, 2005.

[8] A.K. Sharma, "Optimal Number and Location of TCSC and Loadability Enhancement in Deregulated Electricity
Markets Using MINLP”, International Journal of Emerging Electric Power Systems", Vol.5, Issue 1, Article 6, 2006.

[9] S. Gerbex, R. Cherkaoui, and A. J. Germond, "Optimal location of FACTS devices to enhance power system security", 2003 IEEE Bologna PowerTech (IEEE Cat. No.03EX719), Vol.3:7 pp. Vol.3 -, Bologna, Italy, June 23-26, 2003.

[10] L. Cai et al. "Optimal Choice and Allocation of FACTS Devices in Deregulated Electricity Market Using Genetic Algorithms", Power Systems Conference and Exposition, Vol. 1, pp. 201-207, 10-13 Oct. 2004.

[11] L. Ippolito, "Optimal Allocation of FACTS Devices by Using Multi-Objective Optimal Power Flow and Genetic Algorithms", International Journal of Emerging Electric Power Systems, Vol. 7, Issue 2, Article 1, 2006.

[12] Sara Mollazei et al. "Multi-objective Optimization of Power System Performance with TCSC using the MOPSO algorithm", IEEE Power Engineering Society General Meeting, Tampa, Florida. USA. 24-28 June 2007.

[13] M.A. Abido, and J.M. Bakhashwain, "Optimal VAR dispatch using a multiobjective evolutionary algorithm", Electrical Power and Energy Systems, Vol. 27, 2005, pp. 13-20.

[14] T. Ray, and K. Liew, "A swarm metaphor for multiobjective design optimization," Engineering Optimization, Vol. 34, pp. 141-153, 2002.

[15] X. Hu, and R. Eberhart, "Multiobjective optimization using dynamic neighborhood particle swarm optimization," Congress on Evolutionary Computation IEEE Service Center, Piscataway, New Jersey 2002, pp. 1677-1681.

[16] X. Hu, R.C. Eberhart, and Y. Shi, "Particle swarm with extended memory for multiobjective optimization," Proc. IEEE Swarm Intelligence Symposium, IEEE Service Center, Indianapolis, Indiana 2003, pp. 193-197.

[17] C.A. Coello Coello, and M. Salazar Lechuga, "MOPSO: A proposal for multiple objective particle swarm optimization," Congress on Evolutionary Computation IEEE Service Center, Piscataway, New Jersey 2002, pp. 1051-1056.

[18] J. E. Fieldsend, and S. Singh, “A multi-objective algorithm based upon particle swarm optimization, an efficient data structure and turbulence," Proc. of the Workshop on Computational Intelligence, Birmingham, UK 2002, pp. 37-44.

[19] S. Mostaghim, and J. Teich, "Strategies for finding good local guides in multi-objective particle swarm optimization (MOPSO)," Proc. IEEE Swarm Intelligence Symposium, IEEE Service Center, Indianapolis, Indiana 2003, pp. 2633.

[20] X. Li, "A non-dominated Sorting Particle Swarm Optimizer for multiobjective optimization, Proceedings of Genetic and Evolutionary Computation," In SpringerVerlag Lecture Notes in Computer Science, 2003, 2723, pp. 37-48.

[21] Y. Shi, and R. Eberhart, "A Modified Particle Swarm Optimizer," Proc. of IEEE International Conference on Evolutionnary Computation, pp. 69-73, Anchorage, May 1998.

[22] A. Oudalov, "Coordinated Control of Multiple FACTS Devices in an Electric Power System," PHD dissertation, Dept. Electricité, Ecole Polytechnique Fédérale de Lausanne, 2003.

[23] PSAT Version 1.3.4, Software and Documentation, copyright (C) 2002-2005 Federico Milano, July, 2005. 